\title{
Laser-plasma accelerator-based single-cycle attosecond undulator source
}

\author{
Z. Tibai ${ }^{1} \cdot$ Gy. Tóth $^{2} \cdot$ A. Nagyváradi ${ }^{3} \cdot$ A. Sharma ${ }^{4}$ M. I. Mechler ${ }^{2}$ J. A. Fülöp ${ }^{2,5} \cdot$ G. Almási ${ }^{1,2} \cdot$ J. Hebling $^{1,2,5}$
}

Received: 18 August 2017 / Accepted: 8 May 2018 / Published online: 25 May 2018

(c) The Author(s) 2018

\begin{abstract}
Laser-plasma accelerators (LPAs), producing high-quality electron beams, provide an opportunity to reduce the size of free-electron lasers (FELs) to only a few meters. A complete system is proposed here, which is based on FEL technology and consists of an LPA, two undulators, and other magnetic devices. The system is capable to generate carrier-envelope phase stable attosecond pulses with engineered waveform. Pulses with up to $60 \mathrm{~nJ}$ energy and 90-400 attosecond duration in the $30-120 \mathrm{~nm}$ wavelength range are predicted by numerical simulation. These pulses can be used to investigate ultrafast field-driven electron dynamics in matter.
\end{abstract}

\section{Introduction}

The time-resolved study of electron dynamics in atoms, molecules, and solids requires attosecond-scale temporal resolution [1-5]. A suitable tool is provided by isolated attosecond pulses in the extreme ultraviolet (EUV) spectral range, produced by high-harmonic generation (HHG) of waveformcontrolled few-cycle laser pulses. The recent demonstration of single-cycle isolated attosecond pulses [5] may open the way to a new regime in ultrafast physics, where the strongfield electron dynamics is driven by the electric field of the attosecond pulses rather than by their intensity profile. Intense EUV pulses can also be generated in free-electron lasers (FELs), which are expensive large-scale facilities relying on linear accelerators (LINACs). In recent years, many ideas have been proposed to achieve ultrashort pulses in FELs [6-11]. However, the limited temporal coherence

\section{Z. Tibai}

tibai@fizika.ttk.pte.hu

1 Institute of Physics, University of Pécs, Pecs 7624, Hungary

2 MTA-PTE High Field Terahertz Research Group, Pecs 7624, Hungary

3 Faculty of Engineering and Information Technology, University of Pécs, Pecs 7624, Hungary

4 ELI-ALPS, Szeged 6720, Hungary

5 Szentágothai Research Centre, University of Pécs, Pecs 7624, Hungary of the radiation remained a serious drawback for many applications.

Laser-plasma based electron accelerators (LPAs) [12-21] can be a cost-effective alternative for large-scale LINACs, allowing a tremendous reduction of the accelerator size. It is possible to reach electron energies up to the $\mathrm{GeV}$ level over acceleration distances of only a few $\mathrm{cm}$ in a wakefield LPA with parameters comparable to (and in some aspects even better than) conventional LINAC sources [17, 18, 22]. The transversal size of the electron bunch is two orders of magnitude smaller $(\sim 1 \mu \mathrm{m})$ in case of the LPA. However, for the performance of the scheme, the emittance is more important, which is similar in both cases. The peak current in an LPA can be one order of magnitude higher than in a conventional LINAC. Such unique properties of LPA electron pulses offer the potential to construct FELs with a significantly reduced overall dimension and cost, thereby enabling a widespread proliferation of FEL sources to small-scale university labs. Owing to these advantages, numerous schemes have been proposed to use LPA electrons to drive FEL sources [23-30].

Remarkably, none of the previously mentioned techniques, whether HHG-, LPA-, or LINAC-based, could so far produce waveform-engineered CEP-controlled attosecond pulses. Therefore, previously, we proposed a robust method for producing such EUV pulses using conventional LINACbased FEL technology [31-33]. Motivated by the exciting recent progresses in high-power lasers and LPAs delivering high-quality electron beams, here, we investigate the feasibility and the prospects of adopting our previous concept to be driven by an LPA, rather than an LINAC. A detailed 
conceptual study is presented, based on numerical simulations, for an entirely laser-driven waveform-engineered CEPcontrolled attosecond pulse source.

\section{The proposed setup}

The proposed setup for an LPA-based waveform-engineered attosecond pulse source is shown in Fig. 1. Laser pulses of at least $100 \mathrm{TW}$-power are split into two separate beams. The main part is focused into a gas-filled capillary for producing a relativistic electron beam $[17,22]$. After the LPA, the electron beam is sent through the first triplet of quadrupoles to reduce its divergence. Subsequently, a chicane reduces the slice energy spread of the beam. The following second triplet of quadrupoles is used to focus the electron beam into the radiator undulator (RU), which is placed further downstream in the setup. The second quadrupole triplet is followed by the modulator undulator (MU), where the electron bunch is overlapped with the smaller, 20-TW portion of the laser beam. Here, the interaction between the electrons, the magnetic field of the MU, and the electromagnetic field of the modulator laser introduces a spatially periodic energy modulation of the electrons. The electrons propagate through a second chicane and their energy modulation leads to the formation of a train of nanobunches (ultrathin electron layers), separated by the modulator laser wavelength. (The second chicane is used to reduce the propagation distance, where the shortest nanobunches are formed.) The nanobunched electron beam then passes through the RU, consisting of a single or a few periods, and creates CEP-stable attosecond pulses.

\section{Transport and manipulation of the electron beam}

The general particle tracer (GPT) numerical code [34] was used for the simulation of the electron beam transport from the capillary to the RU. Due to limitations of computational capacity, macroparticles consisting of about 4100 electrons were considered, rather than individual electrons. The initial electron bunch parameters are chosen according to feasible parameters for LPA-generated electron beams $[17,22]$ and are listed in Table 1 . We performed a series of simulation runs, during which the electron bunch length was kept constant for each run, but the initial spatial distribution of the electrons was random. We note that particle-in-cell (PIC) simulations [35] can be used to implement more detailed predictions on the initial electron beam parameters, which then can serve as input for the particle-tracking calculations. However, this is out of scope for the present work aiming at assessing the feasibility of the LPA-based CEP-stable attosecond source, rather than giving a complete in-depth numerical design and optimization study. Efficient generation of radiation is possible only if the (micro- or nano) bunch length is shorter than half of the radiation wavelength. If the Coulomb interaction between the electrons in the bunch can be neglected, the following analytical formula can be derived for the FWHM of the electron density of the micro- or nanobunch:

$\Delta b_{0}=\frac{\lambda_{l} \sigma_{\gamma}}{2 \Delta \gamma_{\mathrm{MU}}}$,

where $\lambda_{l}$ is the wavelength of the modulator laser, $\sigma_{\gamma}$ is the energy spread of the nanobunch, and $\Delta \gamma_{\mathrm{MU}}$ is the induced energy gain in the MU $[36,37]$. According to this equation, shorter nanobunches can be achieved using an electron beam with smaller energy spread and stronger energy modulation.

The energy spread of an LPA-generated electron beam is typically much larger $(1-10 \%)$ than that of an LINAC (typically $<0.05 \%$ ). Therefore, a reduction of the slice energy spread is necessary, which can be accomplished by the stretch of the beam or utilizing a transverse-gradient

Table 1 Parameters of the electron beam originating from an LPA, used as initial values in the simulations

\begin{tabular}{ll}
\hline Parameter & Value \\
\hline Energy $\left(\gamma_{0}\right)$ & 2000 \\
Energy spread $\left(\sigma_{\gamma_{0}}\right)$ & $1.0 \%$ \\
Normalized emittance $\left(\gamma_{0} \varepsilon_{x, y}\right)$ & $1.0 \mathrm{~mm} \mathrm{mrad}$ \\
Transversal size $\left(\sigma_{x 0}=\sigma_{y 0}\right)$ & $1.0, \mu \mathrm{m}$ \\
Length $\left(\sigma_{z 0}\right)$ & $1.0 \mu \mathrm{m}$ \\
Charge & $50 \mathrm{pC}$ \\
\hline
\end{tabular}

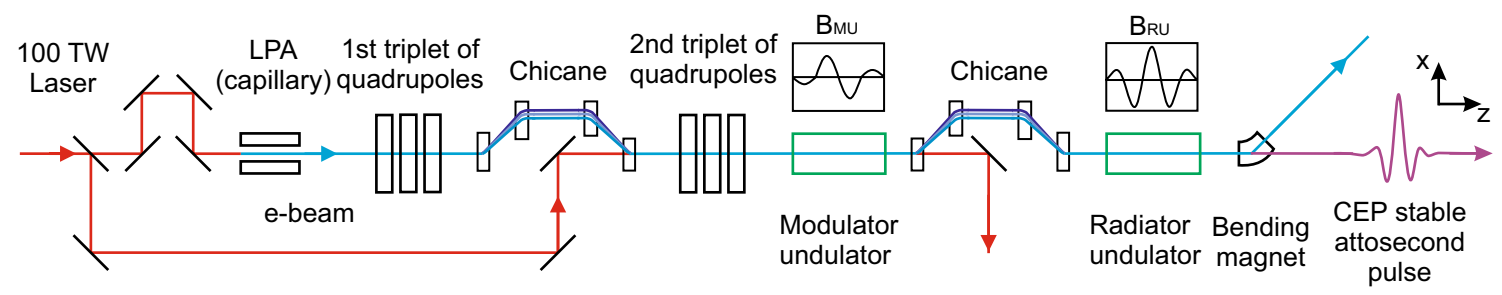

Fig. 1 Scheme of the proposed LPA-based setup 
undulator (TGU) as a radiator [38]. In our setup, we used the first chicane to decompress the beam longitudinally. The chicane consisted of four identical dipole magnets with parameters, as listed in Table 2. The slice energy spread of the electron beam is reduced at the end of the chicane according to the formula [39]:

$\sigma_{\gamma z}=\sigma_{\gamma 0} \frac{\sigma_{z 0}}{\sigma_{z z}}=\sigma_{\gamma 0} \frac{\sigma_{z 0}}{\sqrt{\sigma_{z 0}^{2}+\left(R_{56} \frac{\sigma_{\gamma 0}}{\gamma_{0}}\right)^{2}}}$,

where $\sigma_{z z}$ is the length of the electron beam after the chicane, $R_{56}=2 \theta_{0}^{2}\left(D+2 / 3 L_{\text {mag }}\right), D$ is the distance between $1 \mathrm{st}$ (3rd) and 2nd (4th) dipoles, $L_{\text {mag }}$ is the length of the dipoles, and $\theta_{0}$ is the deviation angle at the dipoles. Hence, the electron bunch can be easily lengthened by adjusting the chicane strength $\left(R_{56}\right)$. The slice energy spread of the beam is reduced from 1 to $0.1 \%$ with $R_{56}=1 \mathrm{~mm}$, and the bunch is lengthened to about $10 \mu \mathrm{m}$ (33 fs).

The modulator laser pulses are used for driving the nanobunch formation. As mentioned in the previous section, these laser pulses are provided by splitting off a small portion of the main beam. Our setup is very advantageous, because one common laser source creates the electron beam and the modulator laser beam, too. Therefore, the synchronization between the electron beam and the modulator laser pulses can be easily solved. Today, numerous 100-TW class laser systems are operating all around the world. For the split-off modulator laser pulses, $20 \mathrm{TW}$ peak power was assumed (Table 3 ). The MU period $\lambda_{M U}$ was chosen to satisfy the well-known resonance condition [40] at $K_{\mathrm{MU}}=2.0$, where $K_{\mathrm{MU}}=\left(e B_{\mathrm{MU}} \lambda_{\mathrm{MU}}\right) /\left(2 \pi m_{e} c\right)$. Here, $e$ is the electron charge, $B_{\mathrm{MU}}$ is the magnetic field amplitude, $m_{e}$ is the electron rest mass, and $c$ is the speed of light. A double-period MU was assumed with antisymmetric design of $-1 / 4,3$ / 4, $-3 / 4$, and $1 / 4$ relative magnetic field amplitudes. According to our calculations, the maximum energy modulation in the MU is $\Delta \gamma_{\mathrm{MU}} \approx 140$. We note that synchrotron radiation (SR) in the chicane and the modulator undulator can increase the energy spread of the bunch. However, according to our calculation (using Ref. [41]), the energy modulation due to SR is four orders of magnitude smaller than

Table 2 Parameters of the first chicane

\begin{tabular}{ll}
\hline Parameter & Value \\
\hline Dipole length $\left(L_{\text {mag }}\right)$ & $15 \mathrm{~cm}$ \\
Gap & $1 \mathrm{~cm}$ \\
Dipole strength & $0.8 \mathrm{~T}$ \\
Distance between 1st & $30 \mathrm{~cm}$ \\
(3rd) and 2nd (4th) & \\
$\quad$ dipoles $(D)$ & \\
Distance between 2nd & $20 \mathrm{~cm}$ \\
$\quad$ and 3rd dipoles $(L)$ & \\
\hline
\end{tabular}

that induced by the laser. The location of the temporal focal point (where the nanobunch length becomes the shortest) was controlled with the second chicane. The total charge in a single nanobunch is about $\sim 0.6 \mathrm{pC}$ and its length is as short as $27 \mathrm{~nm}$ (FWHM). According to our calculation, the length of the nanobunch is increased by $11 \%$ when the space charge effect was also taken into account. The corresponding reduction in the energy of the generated attosecond pulse is less than $4 \%$. Thus, the space charge effect is negligible in our case. The RU was placed around the temporal focal point. The transversal focusing of the electron beam at the position of the RU was achieved with the two permanent-magnet quadrupole triplets with parameters, as shown in Table 4. The gradients of them were optimized with GPT and a selfdeveloped code. The calculated variations of transversal and longitudinal sizes of the whole electron bunch along the propagation direction are shown in Fig. 2. According to our calculations, the transversal size of the electron bunch at the RU in the $x$ and $y$ directions is 42 and $68 \mu \mathrm{m}$, respectively. As shown in Fig. 2, the variation of the transversal size of the bunch inside the RU is negligible.

\section{Single-cycle attosecond pulse generation}

The temporal shape of the attosecond EUV pulses emitted by the extremely short nanobunches in the RU was calculated at a plane positioned $8 \mathrm{~m}$ behind the RU center. EUV

Table 3 Parameters of the modulator laser and the MU

\begin{tabular}{ll}
\hline Parameter & Value \\
\hline Laser wavelength $\left(\lambda_{l}\right)$ & $800 \mathrm{~nm}$ \\
Laser peak power & $20 \mathrm{TW}$ \\
Laser beam waist & $1 \mathrm{~mm}$ \\
Laser beam Rayleigh length & $3.9 \mathrm{~m}$ \\
MU undulator parameter $\left(K_{\mathrm{MU}}\right)$ & 2.0 \\
MU period length $\left(\lambda_{\mathrm{MU}}\right)$ & $2.15 \mathrm{~m}$ \\
MU magnetic field amplitude $\left(B_{\mathrm{MU}}\right)$ & $0.01 \mathrm{~T}$ \\
\hline
\end{tabular}

Table 4 Parameters of the quadrupoles. Three different values were used for $K_{\mathrm{RU}}$, and correspondingly, for $\lambda_{\mathrm{RU}}$

\begin{tabular}{ll}
\hline Parameters & Value \\
\hline $\begin{array}{l}\text { Distance between the capillary and the } 1^{\text {st }} \\
\quad \text { quadrupole }\end{array}$ & $15 \mathrm{~cm}$ \\
1st quadrupole triplet gradients & $197 / 177 / 73 \mathrm{~T} / \mathrm{m}$ \\
1st chicane dipole field & $0.8 \mathrm{~T}$ \\
2nd quadrupole triplet gradients & $47 / 51 / 6 \mathrm{~T} / \mathrm{m}$ \\
2nd chicane dipole field & $0.011 \mathrm{~T}$ \\
RU undulator parameter $\left(K_{\mathrm{RU}}\right)$ & $0.5,0.8$, and 1.2 \\
RU undulator period length $\left(\lambda_{\mathrm{RU}}\right)$ & 43,36, and $28 \mathrm{~cm}$ \\
\hline
\end{tabular}




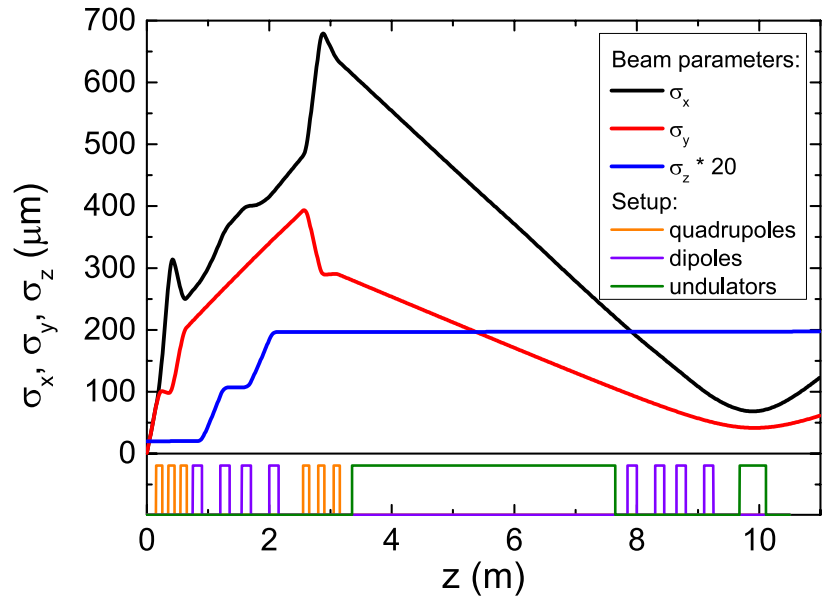

Fig. 2 Variation of the transversal and longitudinal electron bunch sizes along the propagation through the magnetic components of the setup. The size and position of each component are indicated in the bottom part of the figure

waveform engineering is conveniently enabled by designing the magnetic field distribution of the RU. In the present case, the magnetic field of the RU was defined as

$B_{\mathrm{RU}}= \begin{cases}B_{\mathrm{RU} 0} \mathrm{e}^{-\frac{z^{2}}{2 \sigma^{2}}} \cos \left(\frac{2 \pi}{\lambda_{\mathrm{RU}}} z+\varphi\right), & \text { if }-\frac{W}{2}<z<\frac{W}{2}, \\ 0 & \text { otherwise }\end{cases}$

where $B_{\mathrm{RU}}$ is the peak magnetic field, $z$ is the length along the direction of the nanobunch propagation, $\sigma$ is the standard deviation of the Gaussian envelope, $\varphi$ is the CEP, and $W$ is the length of the RU. In each simulation series, the undulator period was adjusted to give the desired central wavelength for the radiation $(20,30,40,60,80,100$, and $120 \mathrm{~nm})$, according to the resonance equation. The further undulator parameters were set to $\sigma=1.5 \times \lambda_{\mathrm{RU}}, W=2.5 \times \lambda_{\mathrm{RU}}$, and $\varphi=0$. (Similar magnetic field distribution has been demonstrated in the experiment of Kimura et al. [42].)

We used the following handbook formula to calculate the electric field of the radiation generated in the RU [43]:
$\mathbf{E}(t, \mathbf{r})=\sum\left[\frac{q \mu_{0}}{4 \pi} \frac{\mathbf{R} \times((\mathbf{R}-R \boldsymbol{\beta}) \times \dot{\mathbf{v}})}{(R-\mathbf{R} \cdot \boldsymbol{\beta})^{3}}\right]_{\mathrm{ret}}$,

where $\mu_{0}$ is the vacuum permeability, $q$ is the macroparticle charge, $\mathbf{R}$ is the vector pointing from the position of the macroparticle at the retarded moment to the observation point, $\mathbf{v}$ is the velocity of the macroparticle, $\boldsymbol{\beta}=\mathbf{v} / c$, and $c$ is the speed of light. The summation is for all macroparticles. During the radiation process, the position, velocity, and acceleration of the macroparticles were traced numerically by taking into account the Lorentz force of the magnetic field of RU. The Coulomb interaction between the macroparticles was neglected during the undulator radiation process, because the transversal electron motion is by four orders of magnitude larger than the motion generated by Coulomb interaction [31].

Figure 3a displays one example of the simulated waveform of the generated attosecond pulse (blue curve) and Fig. 3b displays the corresponding beam profile for $60 \mathrm{~nm}$ radiation wavelength and $K_{\mathrm{RU}}=0.5$ (the cross symbol marks the location, where the waveform in Fig. 3a was sampled). As it is shown in Fig. 3a, the waveform of the generated attosecond pulse resembles the magnetic field of the RU (red curve) [31]. At other wavelengths, the shapes of the attosecond pulses are nearly identical to the shape, as shown in Fig. 3a (blue curve). Besides the CEP stability of the EUV pulses, another important advantage of this setup is that the EUV pulse CEP can be controlled (set) by the magnetic field distribution of the RU [44]. Consequently, attosecond pulses with both single- and multi-cycle waveform can be generated with this technique.

The EUV pulse energy as a function of the radiation wavelength $\left(\lambda_{\mathrm{r}}\right)$ in the range of $20-120 \mathrm{~nm}$ is shown in Fig. $4 \mathrm{a}$, with three different RU undulator parameter values of $0.5,0.8$, and 1.2. These values correspond to 13,24 , and $46 \mathrm{mT}$ peak magnetic fields for $\lambda_{\mathrm{r}}=60 \mathrm{~nm}$. Larger EUV pulse energy is obtained with larger $K_{\mathrm{RU}}$, because the energy of the pulse is proportional to the square of $K_{\mathrm{RU}}$ value. Figure $4 \mathrm{a}$ contains the results of about ten numerical simulation
Fig. 3 a Example of a CEPcontrolled EUV waveform for $60 \mathrm{~nm}$ radiation wavelength and $K_{\mathrm{RU}}=0.5$. b Corresponding spatial beam profile
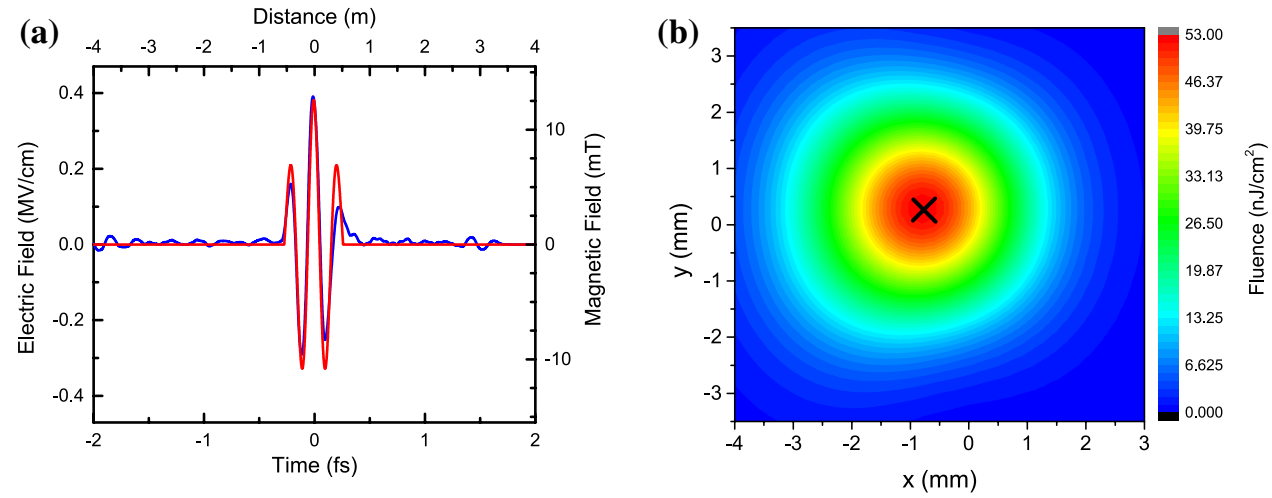
Fig. 4 EUV pulse energy (a) and the period of the RU (b) as functions of the radiation wavelength $\lambda_{\mathrm{r}}$ for different RU undulator parameters
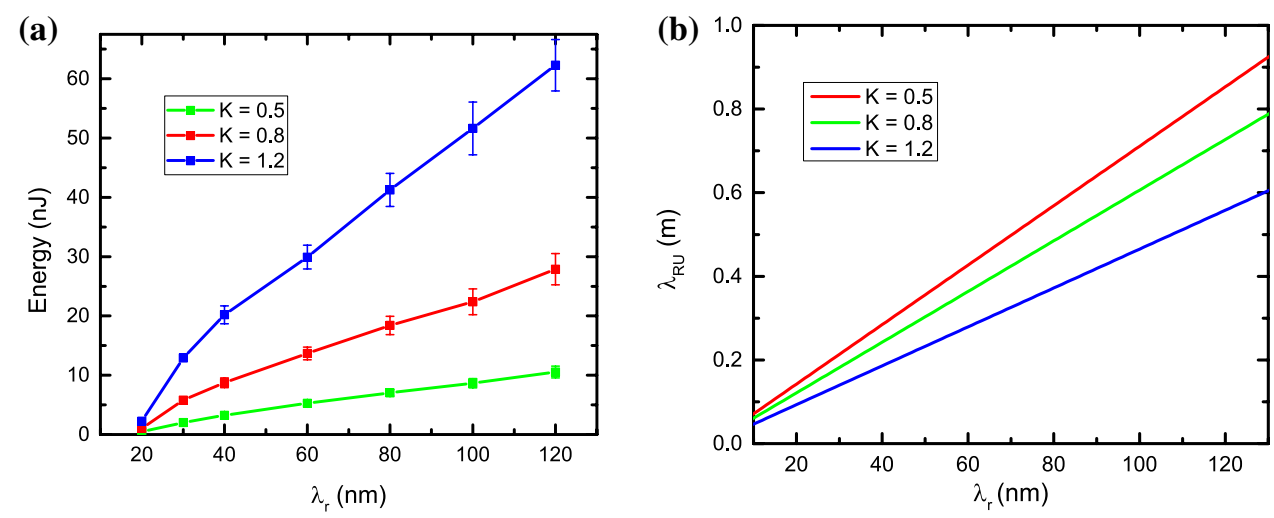

runs for every parameter set, determining also the error bars. The undulator parameter can be set to the desired value by adjusting the magnetic field amplitude. The radiation wavelength, given by the resonance condition, can be set by the choice of the RU period $\lambda_{\mathrm{RU}}$. The period of the RU as a function of the radiation wavelength $\lambda_{\mathrm{r}}$ for different RU undulator parameters is shown in Fig. 4b. Our simulations predict attosecond pulses with up to 10,25 , and $60 \mathrm{~nJ}$ energy for undulator parameters of $0.5,0.8$, and 1.2 , respectively (Fig. 4a). These energies are sufficient for many applications. Importantly, the LPA-based scheme does not require a large-scale accelerator facility and can be affordable for smaller laboratories.

\section{Conclusion}

A robust method for the efficient generation of CEP-stable single-cycle attosecond pulses was proposed, which utilizes a laser-plasma-based electron accelerator, a modulator undulator, and a radiator undulator. The laser pulses used to drive the electron source and the nanobunching can be derived from the same laser, easily enabling the precise synchronization between the electrons and the modulating laser field. The waveform of the attosecond pulses can be engineered by the choice of the magnetic field distribution in the radiator undulator. A conceptual design study was presented, including also a combination of magnetic devices for electron beam transport and manipulation. The generation of single-cycle attosecond pulses in the EUV spectral range with up to $60 \mathrm{~nJ}$ energy was predicted by numerical simulations. The results clearly show that the previously proposed LINAC-based scheme can be adopted to an entirely laser-driven one, thereby enabling to shrink the size of the system from hundreds-of-meters to only a few meters, and a cost-effective implementation in small-scale laboratories. The source is suitable to deliver pump pulses in pump-probe measurements and in time- and CEP-resolved measurements with $\sim 100$-as resolution.
Acknowledgements This work is partially supported by European Cluster of Advanced Laser Light Sources (EUCALL) project which has received funding from the European Union's Horizon 2020 research and innovation programme under grant agreement no. 654220. Financial support from Hungarian Scientific Research Fund (OTKA) Grant No. 113083 is acknowledged. JAF acknowledges support from János Bolyai Research Scholarship (Hungarian Academy of Sciences). The present scientific contribution is dedicated to the $650^{\text {th }}$ anniversary of the foundation of University of Pécs, Hungary. The project has been supported by the European Union, co-financed by the European Social Fund Grant no.: EFOP-3.6.1.-16-2016-00004 entitled by Comprehensive Development for Implementing Smart Specialization Strategies at the University of Pécs; EFOP-3.6.2-16-2017-00005 entitled by Ultrafast physical processes in atoms, molecules, nanostructures and biology structures.

Open Access This article is distributed under the terms of the Creative Commons Attribution 4.0 International License (http://creativeco mmons.org/licenses/by/4.0/), which permits unrestricted use, distribution, and reproduction in any medium, provided you give appropriate credit to the original author(s) and the source, provide a link to the Creative Commons license, and indicate if changes were made.

\section{References}

1. M. Hentschel, R. Kienberger, Ch. Spielmann, G.A. Reider, N. Milosevic, T. Brabec, P. Corkum, U. Heinzmann, M. Drescher, F. Krausz, Attosecond metrology. Nature 414(6863), 509-513 (2001)

2. R. Kienberger, E. Goulielmakis, M. Uiberacker, A. Baltuska, V. Yakovlev, F. Bammer, A. Scrinzi, Th Westerwalbesloh, U. Kleineberg, U. Heinzmann, M. Drescher, F. Krausz, Atomic transient recorder. Nature 427(6977), 817-821 (2004)

3. K.J. Schafer, M.B. Gaarde, A. Heinrich, J. Biegert, U. Keller, Strong field quantum path control using attosecond pulse trains. Phys. Rev. Lett. 92, 023003 (2004)

4. P. Johnsson, R. López-Martens, S. Kazamias, J. Mauritsson, C. Valentin, T. Remetter, K. Varjú, M.B. Gaarde, Y. Mairesse, H. Wabnitz, P. Salières, Ph Balcou, K.J. Schafer, A. L'Huillier, Attosecond electron wave packet dynamics in strong laser fields. Phys. Rev. Lett. 95, 013001 (2005)

5. F. Krausz, M. Ivanov, Attosecond physics. Rev. Mod. Phys. 81, 163-234 (2009)

6. P. Emma, K. Bane, M. Cornacchia, Z. Huang, H. Schlarb, G. Stupakov, D. Walz, Femtosecond and subfemtosecond X-ray pulses 
from a self-amplified spontaneous-emission-based free-electron laser. Phys. Rev. Lett. 92, 074801 (2004)

7. A.A. Zholents, G. Penn, Obtaining attosecond X-ray pulses using a self-amplified spontaneous emission free electron laser. Phys. Rev. ST Accel. Beams 8, 050704 (2005)

8. D.J. Dunning, B.W.J. McNeil, N.R. Thompson, Few-cycle pulse generation in an X-ray free-electron laser. Phys. Rev. Lett. 110, 104801 (2013)

9. B. Garcia, E. Hemsing, T. Raubenheimer, L.T. Campbell, B.W.J. McNeil, Method to generate a pulse train of few-cycle coherent radiation. Phys. Rev. Accel. Beams 19, 090701 (2016)

10. T. Tanaka, Proposal to generate an isolated monocycle X-ray pulse by counteracting the slippage effect in free-electron lasers. Phys. Rev. Lett. 114, 044801 (2015)

11. G. Sansone, E. Benedetti, F. Calegari, C. Vozzi, L. Avaldi, R. Flammini, L. Poletto, P. Villoresi, C. Altucci, R. Velotta, S. Stagira, S. De Silvestri, M. Nisoli, Isolated single-cycle attosecond pulses. Science 314(5798), 443-446 (2006)

12. T. Tajima, J.M. Dawson, Laser electron accelerator. Phys. Rev. Lett. 43, 267-270 (1979)

13. S.P.D. Mangles, C.D. Murphy, Z. Najmudin, A.G.R. Thomas, J.L. Collier, A.E. Dangor, E.J. Divall, P.S. Foster, J.G. Gallacher, C.J. Hooker, D.A. Jaroszynski, A.J. Langley, W.B. Mori, P.A. Norreys, F.S. Tsung, R. Viskup, B.R. Walton, K. Krushelnick, Monoenergetic beams of relativistic electrons from intense laser-plasma interactions. Nature 431(7008), 535-538 (2004)

14. C.G.R. Geddes, C.S. Toth, J. van Tilborg, E. Esarey, C.B. Schroeder, D. Bruhwiler, C. Nieter, J. Cary, W.P. Leemans, Highquality electron beams from a laser wakefield accelerator using plasma-channel guiding. Nature 431(7008), 538-541 (2004)

15. J. Faure, Y. Glinec, A. Pukhov, S. Kiselev, S. Gordienko, E. Lefebvre, J.-P. Rousseau, F. Burgy, V. Malka, A laser-plasma accelerator producing monoenergetic electron beams. Nature 431(7008), 541-544 (2004)

16. V. Malka, J. Faure, Y.A. Gauduel, E. Lefebvre, A. Rousse, K.T. Phuoc, Principles and applications of compact laser-plasma accelerators. Nat. Phys. 4(6), 447-453 (2008)

17. E. Esarey, C.B. Schroeder, W.P. Leemans, Physics of laser-driven plasma-based electron accelerators. Rev. Mod. Phys. 81, 12291285 (2009)

18. X. Wang, R. Zgadzaj, N. Fazel, Z. Li, S.A. Yi, X. Zhang, W. Henderson, Y.Y. Chang, R. Korzekwa, H.E. Tsai, C.H. Pai, H. Quevedo, G. Dyer, E. Gaul, M. Martinez, A.C. Bernstein, T. Borger, M. Spinks, M. Donovan, V. Khudik, G. Shvets, T. Ditmire, M.C. Downer, Quasi-monoenergetic laser-plasma acceleration of electrons to 2 gev. Nat. Commun. 4, 1988 (2013)

19. S.G. Rykovanov, C.B. Schroeder, E. Esarey, C.G.R. Geddes, W.P. Leemans, Plasma undulator based on laser excitation of wakefields in a plasma channel. Phys. Rev. Lett. 114, 145003 (2015)

20. S.G. Rykovanov, J.W. Wang, VYu. Kharin, B. Lei, C.B. Schroeder, C.G.R. Geddes, E. Esarey, W.P. Leemans, Tunable polarization plasma channel undulator for narrow bandwidth photon emission. Phys. Rev. Accel. Beams 19, 090703 (2016)

21. J.W. Wang, C.B. Schroeder, R. Li, M. Zepf, S.G. Rykovanov, Plasma channel undulator excited by high-order laser modes. Sci. Rep. 7(1), 16884 (2017)

22. W.P. Leemans, B. Nagler, A.J. Gonsalves, C.S. Toth, K. Nakamura, C.G.R. Geddes, E. Esarey, C.B. Schroeder, S.M. Hooker, Gev electron beams from a centimetre-scale accelerator. Nat. Phys. 2(10), 696-699 (2006)

23. M.E. Couprie, M. Labat, C. Evain, F. Marteau, F. Briquez, M. Khojoyan, C. Benabderrahmane, L. Chapuis, N. Hubert, C. Bourassin-Bouchet, M. El Ajjouri, F. Bouvet, Y. Dietrich, M. Valléau, G. Sharma, W. Yang, O. Marcouillé, J. Vétéran, P. Berteaud, T. El Ajjouri, L. Cassinari, C. Thaury, G. Lambert, I.
Andriyash, V. Malka, X. Davoine, M.A. Tordeux, C. Miron, D. Zerbib, K. Tavakoli, J.L. Marlats, M. Tilmont, P. Rommeluère, J.P. Duval, M.H. N'Guyen, A. Rouqier, M. Vanderbergue, C. Herbeaux, M. Sebdouai, A. Lestrade, N. Leclercq, D. Dennetière, M. Thomasset, F. Polack, S. Bielawski, C. Szwaj, A. Loulergue, An application of laser-plasma acceleration: towards a free-electron laser amplification. Plasma Phys. Control. Fusion 58(3), 034020 (2016)

24. J. van Tilborg, S.K. Barber, F. Isono, C.B. Schroeder, E. Esarey, W.P. Leemans, Free-electron lasers driven by laser plasma accelerators. AIP Conf. Proc. 1812(1), 020002 (2017)

25. T. Liu, T. Zhang, D. Wang, Z. Huang, Compact beam transport system for free-electron lasers driven by a laser plasma accelerator. Phys. Rev. Accel. Beams 20, 020701 (2017)

26. K. Nakajima, Compact X-ray sources: towards a table-top freeelectron laser. Nat. Phys. 4(2), 92-93 (2008)

27. M. Fuchs, R. Weingartner, A. Popp, Z.S. Major, S. Becker, J. Osterhoff, I. Cortrie, B. Zeitler, R. Horlein, G.D. Tsakiris, U. Schramm, T.P. Rowlands-Rees, S.M. Hooker, D. Habs, F. Krausz, S. Karsch, F. Gruner, Laser-driven soft-X-ray undulator source. Nat. Phys. 5(11), 826-829 (2009)

28. M.E. Couprie, A. Loulergue, M. Labat, R. Lehe, V. Malka, Towards a free electron laser based on laser plasma accelerators. J. Phys. B At. Mol. Opt. Phys. 47(23), 234001 (2014)

29. M.E. Couprie, M. Labat, C. Evain, C. Szwaj, S. Bielawski, N. Hubert, C. Benabderrahmane, F. Briquez, L. Chapuis, F. Marteau, M. Valléau, O. Marcouillé, P. Marchand, M. Diop, J.L. Marlats, K. Tavakoli, D. Zerbib, L. Cassinari, F. Bouvet, C. Herbeaux, C. Bourassin-Bouchet, D. Dennetière, F. Polack, A. Lestrade, M. Khojoyan, W. Yang, G. Sharma, P. Morin, A. Loulergue, Strategies towards a compact xuv free electron laser adopted for the lunex5 project. J. Mod. Opt. 63(4), 309-323 (2016)

30. Chao Feng, Dao Xiang, Haixiao Deng, Dazhang Huang, Dong Wang, Zhentang Zhao, Generating intense fully coherent soft $\mathrm{X}$-ray radiation based on a laser-plasma accelerator. Opt. Express 23(11), 14993-15002 (2015)

31. Z. Tibai, G.Y. Tóth, M.I. Mechler, J.A. Fülöp, G. Almási, J. Hebling, Proposal for carrier-envelope-phase stable single-cycle attosecond pulse generation in the extreme-ultraviolet range. Phys. Rev. Lett. 113, 104801 (2014)

32. G.Y. Tóth, Z. Tibai, Z.S. Nagy-Csiha, Z.S. Márton, G. Almási, J. Hebling, Circularly polarized carrier-envelope-phase stable attosecond pulse generation based on coherent undulator radiation. Opt. Lett. 40(18), 4317-4320 (2015)

33. Z. Tibai, G. Tóth, Z. Nagy-Csiha, J.A. Fülöp, G. Almási, $\mathrm{J}$. Hebling, Investigation of the newly proposed carrier-envelopephase stable attosecond pulse source. arXiv:1604.08050 (2016)

34. Online at http://www.pulsar.nl/gpt

35. M. Bussmann, H. Burau, T.E. Cowan, A. Debus, A. Huebl, G. Juckeland, T. Kluge, W.E. Nagel, R. Pausch, F. Schmitt, U. Schramm, J. Schuchart, R. Widera, Radiative signature of the relativistic kelvin-helmholtz instability. In: 2013 SC-International Conference for High Performance Computing, Networking, Storage and Analysis (SC), pp. 1-12 (2013)

36. E. Hemsing, G. Stupakov, D. Xiang, A. Zholents, Beam by design: laser manipulation of electrons in modern accelerators. Rev. Mod. Phys. 86, 897-941 (2014)

37. A.A. Zholents, Method of an enhanced self-amplified spontaneous emission for X-ray free electron lasers. Phys. Rev. ST Accel. Beams 8, 040701 (2005)

38. Z. Huang, Y. Ding, C.B. Schroeder, Compact X-ray free-electron laser from a laser-plasma accelerator using a transverse-gradient undulator. Phys. Rev. Lett. 109, 204801 (2012)

39. M.E. Couprie, C. Benabderrahmane, P. Betinelli, F. Bouvet, A. Buteau, L. Cassinari, J. Daillant, J.C. Denard, P. Eymard, B. 
Gagey, C. Herbeaux, M. Labat, B. Lagarde, A. Lestrade, A. Loulergue, P. Marchand, J.L. Marlats, C. Miron, P. Morin, A. Nadji, F. Polack, J.B. Pruvost, F. Ribeiro, J.P. Ricaud, P. Roy, T. Tanikawa, R. Roux, S. Bielawski, C. Evain, C. Szwaj, G. Lambert, A. Lifschitz, V. Malka, R. Lehe, A. Rousse, K.T. Phuoc, C. Thaury, G. Devanz, M. Luong, B. Carré, G. LeBec, L. Farvacque, A. Dubois, J. Lüning, J. Lüning, The LUNEX5 project in france. J. Phys. Conf. Ser. 425(7), 072001 (2013)

40. H. Wiedemann, Particle Accelerator Physics, Volume I and II of Advanced Texts in Physics (Springer, Berlin, 2003)

41. A. Zholents, G. Penn, Obtaining two attosecond pulses for X-ray stimulated raman spectroscopy. Nucl. Instrum. Methods Phys. Res. A 612, 254-259 (2010)
42. W.D. Kimura, L.P. Campbell, C.E. Dilley, S.C. Gottschalk, D.C. Quimby, M. Babzien, I. Ben-Zvi, J.C. Gallardo, K.P. Kusche, I.V. Pogorelsky, J. Skaritka, V. Yakimenko, D.B. Cline, F. Zhou, L.C. Steinhauer, R.H. Pantell, Detailed experimental results for hightrapping efficiency and narrow energy spread in a laser-driven accelerator. Phys. Rev. ST Accel. Beams 7, 091301 (2004)

43. J.D. Jackson, Classical Electrodynamics (Wiley, Oxford, 2007)

44. G.Y. Tóth, Z. Tibai, Z.S. Nagy-Csiha, Z.S. Márton, G. Almási, J. Hebling, Investigation of novel shape-controlled linearly and circularly polarized attosecond pulse sources. Photon and fast ion induced processes in atoms, molecules and nanostructures (PIPAMON). Nucl. Instrum. Methods Phys. Res. Sect. B 369, 2-8 (2016) 